OPEN ACCESS

Edited by: Jose Renato Pinto,

Florida State University, USA

Reviewed by:

Henry G. Zot,

University of West Georgia, USA

Hyun Seok Hwang,

Florida State University, USA

*Correspondence:

Aldrin V. Gomes

avgomes@ucdavis.edu

Specialty section:

This article was submitted to Striated Muscle Physiology, a section of the journal

Frontiers in Physiology

Received: 01 September 2016 Accepted: 20 October 2016 Published: 15 November 2016

Citation:

Nguyen S, Siu R, Dewey S, Cui Z and Gomes AV (2016) Amino Acid Changes at Arginine 204 of Troponin 1 Result in Increased Calcium Sensitivity of Force Development Front. Physiol. 7:520 doi: 10.3389/fphys.2016.00520

\section{Amino Acid Changes at Arginine 204 of Troponin I Result in Increased Calcium Sensitivity of Force Development}

\author{
Susan Nguyen ${ }^{1}$, Rylie Siu ${ }^{1}$, Shannamar Dewey ${ }^{1}$, Ziyou Cui ${ }^{1}$ and Aldrin V. Gomes ${ }^{1,2 *}$ \\ 1 Department of Neurobiology, Physiology, and Behavior, University of California, Davis, Davis, CA, USA, ${ }^{2}$ Department of \\ Physiology and Membrane Biology, University of California, Davis, Davis, CA, USA
}

Mutations in human cardiac troponin I (cTnl) have been associated with restrictive, dilated, and hypertrophic cardiomyopathies. The most commonly occurring residue on cTnl associated with familial hypertrophic cardiomyopathy (FHC) is arginine (R), which is also the most common residue at which multiple mutations occur. Two FHC mutations are known to occur at cTnl arginine 204, R204C and R204H, and both are associated with poor clinical prognosis. The R204H mutation has also been associated with restrictive cardiomyopathy (RCM). To characterize the effects of different mutations at the same residue (R204) on the physiological function of cTnl, six mutations at R204 (C, G, H, P, Q, W) were investigated in skinned fiber studies. Skinned fiber studies showed that all tested mutations at R204 caused significant increases in $\mathrm{Ca}^{2+}$ sensitivity of force development $\left(\Delta \mathrm{pCa}_{50}=0.22-0.35\right)$ when compared to wild-type (WT) cTnl. Investigation of the interactions between the cTnl mutants and WT cardiac troponin C (cTnC) or WT cardiac troponin T (cTnT) showed that all the mutations investigated, except R204G, affected either or both cTnl:cTnT and cTnl:cTnC interactions. The R204H mutation affected both cTnl:cTnT and cTnl:cTnC interactions while the R204C mutation affected only the cTnl:cTnC interaction. These results suggest that different mutations at the same site on cTnl could have varying effects on thin filament interactions. A mutation in fast skeletal Tnl (R174Q, homologous to cTnl R204Q) also significantly increased $\mathrm{Ca}^{2+}$ sensitivity of force development $\left(\Delta \mathrm{pCa}_{50}=0.16\right)$. Our studies indicate that known $\mathrm{cTnl}$ mutations associated with poor prognosis (R204C and R204H) exhibit large increases in $\mathrm{Ca}^{2+}$ sensitivity of force development. Therefore, other R204 mutations that cause similar increases in $\mathrm{Ca}^{2+}$ sensitivity are also likely to have poor prognoses.

Keywords: troponin I, familial hypertrophic cardiomyopathy, distal arthrogryposis, $\mathrm{Ca}^{2+}$ sensitivity, mammalian two-hybrid

\section{INTRODUCTION}

Familial hypertrophic cardiomyopathy (FHC) is a genetically heterogeneous autosomal dominant inherited heart disease of the myocardium that results in a high incidence of sudden cardiac death and has been linked to mutations in genes coding for at least 10 sarcomeric proteins. These mutations have been found in the genes for: $\alpha$-myosin heavy chain

Abbreviations: FHC, familial hypertrophic cardiomyopathy; HcTnI, human cardiac troponin I; R204, arginine residue 204 on cardiac troponin I; TM, tropomyosin; Tn, troponin; TnC, troponin C; TnI, troponin I; TnT, troponin T; MOPS, 3-[N-morpholino]propanesulonic acid; WT, wild-type. 
(Geisterfer-Lowrance et al., 1990), cardiac myosin essential light chain, and cardiac myosin regulatory light chain (Poetter et al., 1996), $\alpha$-tropomyosin (Thierfelder et al., 1994), cardiac troponin $\mathrm{T}$ (TnT) (Thierfelder et al., 1994), cardiac myosin binding protein C (Bonne et al., 1995; Watkins et al., 1995), cardiac troponin I (TnI) (Kimura et al., 1997), $\alpha$-actin (Mogensen et al., 1999), troponin C (TnC) (Hoffmann et al., 2001), and titin (Satoh et al., 1999). FHC is the most commonly identified cause of sudden death in young adults. Symptoms of FHC vary between individuals, and most patients are asymptomatic (or only exhibit mild symptoms) before sudden cardiac death (O'Mahony and Elliott, 2014).

$\mathrm{TnI}$ is a basic protein that readily interacts with the acidic $\mathrm{TnC}$ and binds with another acidic protein, TnT, to form the Troponin (Tn) complex (Zot and Potter, 1987; Tobacman, 1996; Westfall and Metzger, 2001). TnI is also capable of binding actin and inhibiting $\mathrm{Mg}^{2+}$-activated actomyosin ATPase and is commonly referred to as the inhibitory subunit of Tn. The Tn complex and tropomyosin are critical regulatory components of the muscle contraction apparatus (Xu et al., 2010). Muscle contraction involves cyclic binding and release of cross-bridges (myosin heads of the thick filament interacting with and releasing actin of the thin filament) with energy derived from the hydrolysis of ATP.

Kimura et al. reported five missense mutations in TnI (R145G, R145Q, R162W, G203S, and K206Q) that are linked to FHC (Kimura et al., 1997). Since this initial report, several other TnI FHC mutants [examples include R21C, P82S, K183 deletion ( $\Delta$ K183), I195M, D195N, S199N, R204C, R204H and an exon 8 deletion mutant encompassing the stop codon of the cTnI gene] have also been reported (Kokado et al., 2000; Morner et al., 2000; Niimura et al., 2002; Doolan et al., 2005). FHC mutations in TnI and other myofilament proteins have been previously shown by many groups to generally result in increased $\mathrm{Ca}^{2+}$-sensitivity of force development (Gomes and Potter, 2004). Since R204 had two known mutations (R204C and R204H), and arginine is the amino acid most commonly associated with FHC in TnI or TnT (Xu et al., 2010), it was hypothesized that any mutations at $\mathrm{R} 204$ would cause similar increases in $\mathrm{Ca}^{2+}$-sensitivity of force development. The R204 residue is an important amino acid to investigate since families with the cardiac TnI (cTnI) R204H mutation have a high incidence of sudden death (Doolan et al., 2005). Doolan et al. (2005), investigated R162G, R162P, L198P, $\mathrm{R} 204 \mathrm{H}$ mutations and hypothesized that the FHC phenotype of missense mutations of arginine residues of TnI are due to disrupted functional interactions with $\mathrm{TnC}$ and $\mathrm{TnT}$, which is tested in the present work using multiple residue replacements of R204.

The cTnI R204 residue is well conserved in different animals suggesting that this residue is evolutionarily important. A comparison of the primary amino acid sequence of cTnI and fast skeletal Troponin I (fsTnI) shows that R204 residue corresponds to the R174 residue of fsTnI. It is hypothesized that changes at the fsTnI R174 residue will show similar increases in $\mathrm{Ca}^{2+}$-sensitivity of force development as observed for the cTnI R204 mutants. Since a known missense mutation (R174Q) in fsTnI was shown to be associated with distal arthrogryposis type $2 \mathrm{~B}$ (DA2B, also known as arthrogryposis multiplex congenita, distal, type $2 \mathrm{~B}$ ) (Westfall and Metzger, 2001), this mutation was also investigated in skeletal muscle to determine if this mutation showed similar in vitro physiological properties as the CTnI R204Q mutation. Distal arthrogryposis (DA) refers to a group of disorders characterized by multiple congenital contractures of the hands/wrists and feet/ankles, and affected individuals typically have a triangular shaped face, a small mouth, a prominent chin and positional foot deformities (calcaneovalgus and/or clubfoot).

Investigation of six cTnI R204 residues as well as the fsTnI R174Q mutation showed that all the mutations investigated increased the $\mathrm{Ca}^{2+}$ sensitivity of force development. The different effects of these mutants on the interaction between Tn subunits and the maximal force suggest that the change in $\mathrm{Ca}^{2+}$ sensitivity of force development may be a major determinant for the poor prognosis of patients with the cTnI R204C, R204H, and fsTnI R174Q mutations.

\section{EXPERIMENTAL PROCEDURES}

\section{Mutation, Expression, and Purification of HcTnl and HcTnl Mutants}

The HcTnI FHC mutants were formed by overlapping PCR using HcTnI cDNA obtained from Dr. J.D. Potter (Zhang et al., 1995). Mutation, expression, and purification of $\mathrm{cTnI}$ and TnT mutants were carried out as previously described (Szczesna et al., 2000; Gomes et al., 2005b). The PCR products obtained were digested with NcoI and BamH1 and then ligated to pET-3d vector. The sequence of the TnI mutants was verified by sequencing prior to expression and purification. HcTnI and HcTnI R204 mutants were purified via conventional methods. Briefly, crude bacterial supernatants were purified by column chromatography on an SSepharose column at $4^{\circ} \mathrm{C}$ and eluted with a linear $\mathrm{KCl}$ gradient of $0-0.5 \mathrm{M}$ in a Tris- $\mathrm{HCl}$ buffer containing $6 \mathrm{M}$ urea. Semipure HcTnI and HcTnI mutantswere dialyzed against a solution containing $50 \mathrm{mM}$ Tris- $\mathrm{HCl}, \mathrm{pH}$ 7.5, $1 \mathrm{M} \mathrm{KCl}, 1 \mathrm{M}$ urea, $1 \mathrm{mM}$ DTT, and $2 \mathrm{mM} \mathrm{CaCl}_{2}$ and loaded onto an affinity column having covalently bound HcTnC. Pure HcTnI and HcTnImutants were eluted with a gradient of 0-3 mM EDTA and 1-6 M urea. The purity of the TnI proteins was determined by SDS-PAGE as previously described (Gilda et al., 2016).

\section{Mutation and Expression of Wild-Type and Mutant Fast Skeletal Tnl}

The cDNA encoding rabbit fsTnI was previously cloned by reverse transcriptase-polymerase chain reaction using a template of total RNA from rabbit skeletal muscle and oligonucleotide primers specific for the $5^{\prime}$ and $3^{\prime}$ regions of the respective coding sequences (Sheng et al., 1992). Additionally, the fsTnI R174Q mutant was made using a sequential overlapping polymerase chain reaction-based method as previously described (Gomes et al., 2004, 2005a). Clones were sequenced to verify the correct sequences prior to expression and purification of the respective proteins. 


\section{Protein Purification of Rabbit Fast Skeletal TnT, Tnl, and TnC}

The purification of fsTnI was similar to previously described (Pan and Potter, 1992) with the exception that the final purification step was with TnC-agarose affinity chromatography. Briefly, after S-Sepharose cation exchange chromatography in the presence of $6 \mathrm{M}$, fsTnI was eluted with a continuous ionic strength gradient of $0-0.3 \mathrm{M} \mathrm{NaCl}$. The fsTnI containing fractions were pooled and dialyzed in steps (to remove urea) with $6 \mathrm{M}$ urea, $4 \mathrm{M}$ urea, 2 $\mathrm{M}$ urea in TnC affinity buffer to TnC affinity buffer with no urea (50 mM Tris, pH 7.5 containing $2 \mathrm{mM} \mathrm{CaCl}_{2}, 0.5 \mathrm{M} \mathrm{NaCl}$, and 1 $\mathrm{mM}$ DTT). A column with cardiac TnC immobilized onto $\mathrm{CNBr}-$ activated Sepharose-4B (2 $\mathrm{mg} \mathrm{TnC} / \mathrm{ml}$ agarose) was prepared as described by Pharmacia LKB Biotechnology. The dialyzed TnI was loaded onto the TnC-agarose column equilibrated with TnC affinity buffer. The bound TnI was eluted with TnC elution buffer containing $6 \mathrm{M}$ urea and $3 \mathrm{mM}$ EDTA (50 mM Tris, $0.5 \mathrm{mM}$ $\mathrm{NaCl}, 1 \mathrm{mM}$ DTT, $3 \mathrm{mM}$ EDTA, $6 \mathrm{M}$ urea, $\mathrm{pH}$ 7.5). The eluates were run on SDS gels and purified TnI pooled.

\section{Formation of the Troponin Complex}

Formation of the human cardiac troponin and rabbit skeletal troponin complexes containing recombinant TnT, TnC, and TnI was carried out as recently described by Szczesna et al. (2000), (Szczesna et al., 2000; Gomes et al., 2005b). Proper stoichiometry was verified by SDS-PAGE (Gilda et al., 2016). Although not done routinely, gel filtration of these formed Tn complexes showed that this reconstitution method resulted in a single species.

\section{Preparation of Porcine Skinned Cardiac Muscle Bundles}

Cardiac skinned muscle fibers were prepared following a common laboratory procedure published by Zhang et al. (1995) (Zhang et al., 1995; Gomes et al., 2005a,b). Freshly isolated porcine hearts were incubated in an $\mathrm{O}_{2}$-saturated solution containing $140 \mathrm{mM} \mathrm{NaCl}, 4 \mathrm{mM} \mathrm{KCl}, 1.8 \mathrm{mM} \mathrm{CaCl}_{2}, 1 \mathrm{mM}$ $\mathrm{MgCl}_{2}, 1.8 \mathrm{mM} \mathrm{NaHPO}_{4}, 5.5 \mathrm{mM}$ glucose, and $5 \mathrm{mM}$ HEPES, $\mathrm{pH}$ 7.4. Cardiac muscle bundles were dissected from the left ventricle of the porcine hearts and were chemically skinned by incubating with $50 \%$ glycerol and $1 \%$ Triton $\mathrm{X}-100$ in the relaxing solution (pCa 8.0) containing $10^{-8} \mathrm{M} \mathrm{Ca}^{2+}, 5 \mathrm{mM} \mathrm{Mg}^{2+}, 7 \mathrm{mM}$ EGTA, 20 $\mathrm{mM}$ MgATP, $20 \mathrm{mM}$ creatine phosphate, and $15 \mathrm{U} / \mathrm{mL}$ creatine phosphokinase, $\mathrm{pH} 7.0$, at an ionic strength of $150 \mathrm{mM}$ at $4 \mathrm{C}$ for $24 \mathrm{~h}$. These skinned muscle preparations were dissected into small bundles (1-2 cm in length, 2-3 $\mathrm{mm}$ in diameter) and were stored at $-20^{\circ} \mathrm{C}$ in the same solution without triton $\mathrm{X}-100$ before use.

\section{Steady-State Force and $\mathrm{Ca}^{2+}$-Sensitivity of Force Development of Tn Complexes Containing HcTnl Mutants}

The skinned fiber preparation was mounted with stainless steel clips on a force transducer and was immersed in the contracting solution to measure initial force before treatment. The contraction solution ( $\mathrm{pCa} 4$ ) had the same composition as the relaxation buffer except for the increased $\mathrm{Ca}^{2+}$ concentration $\left(10^{-4} \mathrm{M}\right)$. To determine the $\mathrm{Ca}^{2+}$ dependence of force development, the contraction of the skinned fibers was tested in solutions of intermediate concentrations of $\mathrm{Ca}^{2+}$. The $\mathrm{Ca}^{2+}$ dependence of force was determined before and after performing the displacement and reconstitution protocols that are described below. To remove the endogenous Tn complex, the TnT displacement method was used (Hatakenaka and Ohtsuki, 1992; Szczesna et al., 2000; Gomes et al., 2005a). The cardiac fiber was incubated with HCTnT $(0.8 \mathrm{mg} / \mathrm{mL})$ for a total of 2.5 $\mathrm{h}$ at room temperature with an intermediate buffer change containing fresh HCTnT $(0.8 \mathrm{mg} / \mathrm{mL})$. After displacement of the endogenous complex by HCTnT, the level of unregulated force development was observed by measuring the level of force reached by skinned fibers in both the pCa 8 solution and the pCa 4 solutions. Restoration of the $\mathrm{Ca}^{2+}$ regulation of force development was performed using troponin I:troponin C complexes $(30 \mu \mathrm{m})$ in pCa 8 solution for $\sim 1.5 \mathrm{~h}$ at room temperature. To determine the $\mathrm{Ca}^{2+}$ dependence of force development, the contraction of skinned fibers was tested in solutions with intermediate concentrations of $\mathrm{Ca}^{2+}$ (from $\mathrm{pCa}$ 8 to $\mathrm{pCa} 4$ ). The $\mathrm{Ca}^{2+}$ dependence was determined before and after treatment of the skinned fibers with displacement and reconstitution solutions. The $\mathrm{Ca}^{2+}$ dependence data were analyzed using the Hill equation (Sigmaplot, Jandel Scientific): relative force $(\%)=\left[\mathrm{Ca}^{2+}\right]^{\mathrm{n}} /\left(\left[\mathrm{Ca}^{2+}\right]^{\mathrm{n}}+\left[\mathrm{pCa}_{50}\right]^{\mathrm{n}}\right)$, where $\mathrm{pCa} 50$ is the $\mathrm{pCa}$ of a solution in which $50 \%$ of the change is produced, and $\mathrm{n}$ is the Hill coefficient.

\section{Steady-State Force and the $\mathrm{Ca}^{2+}$-Sensitivity of Force Development of Tn Complexes Containing Wild-Type and FsTnIR174Q}

Skeletal muscle fiber bundles from rabbit psoas muscle were mounted on a force transducer and treated with the pCa 8 relaxing solution containing $1 \%$ Triton $\mathrm{X}-100$ for $\sim 1 \mathrm{~h}$. The composition of the pCa 8 solution was $10^{-8} \mathrm{M}^{2}\left[\mathrm{Ca}^{2+}\right], 1 \mathrm{mM}$ $\left[\mathrm{Mg}^{2+}\right], 7 \mathrm{mM}$ EGTA, $5 \mathrm{mM}\left[\mathrm{MgATp}^{2+}\right], 20 \mathrm{mM}$ imidazole, $\mathrm{pH}$ 7.0, $20 \mathrm{mM}$ creatinine phosphate, and 15 units/ml creatinine phosphokinase, ionic strength $=150 \mathrm{mM}$. To determine the $\mathrm{Ca}^{2+}$ sensitivity of force development, the fibers were gradually exposed to the solutions of increasing $\mathrm{Ca}^{2+}$ concentrations, from pCa 8 to pCa 4 . To displace the endogenous Tn complex from the fibers they were incubated in a solution containing $250 \mathrm{mM}$ KCI, $20 \mathrm{mM}$ MOPS, pH 6.2, $5 \mathrm{mM} \mathrm{MgCl}_{2}, 5 \mathrm{mM}$ EGTA, 0.5 $\mathrm{mM}$ dithiothreitol, and $1.6-1.8 \mathrm{mg} / \mathrm{ml} \mathrm{fsTnT,} \mathrm{for} 1 \mathrm{~h}$ at room temperature. A fresh fsTnT protein was applied to the fibers for another $1 \mathrm{~h}$ incubation. This was to increase the efficiency of the endogenous Tn displacement from the fibers. Displaced fibers were then washed with the same solution without the protein (10 min at room temperature) and tested for $\mathrm{Ca}^{2+}$-unregulated force that developed due to the absence of the endogenous TnI and $\mathrm{TnC}$. The $\mathrm{Ca}^{2+}$ regulation of steady-state force was restored with a preformed fsTnI.fsTnC complex. The reconstitution with the fsTnI:fsTnC complex $(25 \mu \mathrm{M})$ was performed in the pCa 8 solution for $\sim 1.5 \mathrm{~h}$ at room temperature, or long enough for the force to reach a stable level. Control fibers were run in parallel 
and treated with the same solutions minus the proteins. The final $\mathrm{Ca}^{2+}$-sensitivity of force development was determined after fsTnI.fsTnC reconstitution and the data were analyzed with the Hill equation.

\section{Mammalian Two-Hybrid Studies}

Protein:protein interactions were measured using the Checkmate Mammalian Two-Hybrid System (Promega) as previously described (Gilda et al., 2016). cTnI WT and cTnI deletion mutants as well as WT cTnT, and cTnC were subcloned into pACT and pBIND vectors. CV1 cells were transfected with pACT and pBind DNA constructs as well as the pG5luc Vector. TransIT-LT1 transfection reagent (Mirus) was used for all experiments and cells were incubated for 36-48 h following transfection with no media change. Cells were lysed and analyzed using the dual luciferase reporter assay (Promega). To verify the experiments were working correctly, control experiments using various combinations of empty vectors, cTnI, cTnC, or cTnT subcloned vectors were also carried out as previously described (Gilda et al., 2016). To control for potential differential protein expression in the two-hybrid assay, protein levels of the wild-type, and TnI mutants (cloned into the mammalian two hybrid plasmids) in CV1 cells were initially checked by Western blotting to determine if any of the mutants investigated showed significantly different expression levels. All TnI mutants were found to show similar expression levels after $48 \mathrm{~h}$.

\section{Statistical Methods}

All data are presented as mean \pm S.D. Comparisons of mammalian-two hybrid data and maximal force data were carried out using one way ANOVA. Unpaired Student's $t$ test was used to determine the significance of differences in $\triangle \mathrm{pCa}$ (changes in half-maximal activating $\mathrm{pCa}$ in different skinned fibers). Values of $P<0.05$ were considered statistically significant.

\section{RESULTS}

Figure 1A shows the primary structure of the C-terminal region of cTnI with the cTnI FHC mutations that occur within this region indicated. Figure 1B shows the primary sequence alignment for HcTnI and human fsTnI (HfsTnI) indicating that the position of the R204 mutation in HcTnI corresponds to R174 in HfsTnI.

\section{Force Development and the $\mathrm{Ca}^{2+}$ Dependence of Force Development for cTnl Mutations}

To determine the effect of these mutations (R204C and R204H), as well as other potential cTnI mutations, R204G, R204P, R204Q, and R204W, cardiac skinned fiber calcium-force measurements were carried out. We employed a well-established method in our lab (Szczesna et al., 2000; Gomes et al., 2002; Lang et al., 2002) to displace the endogenous Tn complex from skinned porcine cardiac muscle preparations. After determining the level of unregulated force in skinned fibers, they were incubated with either wild-type or mutant HcTnI·HcTnC complexes in low calcium buffer ( $\mathrm{pCa} 8$ ). This allowed us to determine whether the TnI proteins could fully inhibit $\mathrm{Ca}^{2+}$ unregulated force established after treatment with HcTnT and also to determine if the proteins were able to fully reconstitute the skinned fibers by forming a functional Tn complex. Wild-type HcTnI-HcTnC complex resulted in complete inhibition of $\mathrm{Ca}^{2+}$ unregulated force.

All six mutations showed significant increases in calcium sensitivity of force development ranging from $\Delta \mathrm{pCa}_{50} 0.22$ (R204W) to 0.37 (R204G) (Figure 2, Table 1). The mutations associated with FHC, R204C, and R204H, had $\Delta$ pCa50 values of 0.27 and 0.28 respectively (Figure 2, Table 1). Recovered force is equivalent to the level of force developed in fibers after reconstituting the fibers with the appropriate Tn complex. The maximal force (force at pCa 4.0) obtained from the skinned fibers was increased for cTnI R204W, R204C, and R204H mutations relative to wild-type cTnI (Figure 3). It is interesting that both known FHC mutations, R204C and R204H, increase maximal force.

\section{Mammalian Two-Hybrid}

To determine the effect of the R204 mutations on cTnI: cTnT and cTnI: cTnC interactions, the mammalian two-hybrid luciferase assay was utilized. In the CheckMate ${ }^{\mathrm{TM}}$ mammalian two-hybrid system utilized, the pACT vector contains the herpes simplex virus VP16 activation domain upstream of the cloning region, while the pBIND vector contains the yeast GAL4 DNA-binding domain upstream of the cloning region. The pBIND Vector also expresses Renilla reniformis luciferase under the control of the SV40 promoter, which allows the normalization for differences in transfection efficiency. The troponin subunits were cloned into pBIND and pACT Vectors to generate fusion proteins with the DNA-binding domain of GAL4 and the activation domain of VP16, respectively. Association of the DNA-binding domain and the transcriptional activation domain results in transcriptional activation of the firefly luciferase reporter gene and an increase in firefly luciferase expression when compared to the negative controls. Mutations in interacting proteins that disrupt or significantly reduce the interactions between the two proteins being investigated would result in reduced association between the DNA-binding domain and the transcriptional activation domain resulting in less firefly luciferase expression.

The cTnI R204P and R204H mutations showed the weakest interactions with cTnT when compared to wild-type cTnI (Figure 4). The other mutations, including R204C, showed no significant impairment when compared to wild-type cTnI. These results suggest differences in the interactions between R204C and R204H with cTnT. Four of the six mutations investigated (R204Q, R204W, R204C, R204H) showed weaker interactions with cTnC (Figure 5). The cTnI R204P mutation which showed weaker interaction with cTnT, interacted with cTnC similarly to wild-type cTnI (Figure 5). The cTnI R204G mutant also did not show any significant impairment in cTnC binding when compared to wild-type cTnI. The disruption in binding between cTnI and its binding partners may be due to conformation changes in cTnI caused by the mutations. 
A

MCTNI

RBCTNI

HCTNI

RCTNI

BCTNI

HSTNI

RBSTNI

HFTNI

MFTNI

MCTNI

RBCTNI

HCTNI

RCTNI

BCTNI

HSTNI

RBSTNI

HFTNI

MFTNI \begin{tabular}{ccc} 
& \multicolumn{2}{c}{ R145G/Q } \\
130 & 140 & \multicolumn{1}{c}{150}
\end{tabular}
160

KNITEIADLTQKIYDLRGKFKRPTIR-RVRI SADAMMQALLGTRAKES LDLRAHLKOVKK KNITEIADLTQKIYDLRGKFKRPTIR-RVRI SADAMMQALLGT RAKES LDLRAHLKQVKK KNITEIADLTQKIFDLRGKFKRPTIR-RVRI SADAMMQALLGA RAKES LDLRAHLKQVKK KNITEIADLTQKI FDLRGKFKRPT I R RVRI SADAMMQALLGT RAKETLDLRAHLKQVKK KNITEIADLNQKIFDLRGKFKRPTI R-RVRI SADAMMQALLGA PAKETLDLRAHLKQVKK HNTREI KDLKLKVMDLRGKFKRPPI R-RVRVSADAMLRALLGS KKKVSMDLRANLKSVKK HNTREIKDLKLKVLDLRGKFKRP P IR-RVRVSADAMLRALLGS KKKVSMDLRANLKSVKK KT S KELEDMNQKLFDLRGKFKRP P IR-RVRMSADAMLKALLGS KHKVCMDLRANLKQVKK KS S KELEDMNQKLFDLRGKFKRP P I R-RVRMSADAMLKALLGS KHKVCMDLRANLKQVKK

\section{D196N}

$\Delta \mathrm{K} 183$ I195M \S199N G203S R204C

B

HCTNI MADGSSDAAREPRPAPAPIRRRSSNYRAYATEPHAKKKSKISASRKLQLKTLLLQIAKQE 60 HFSTNI MGD----------------------EEKRNRAITARRQHLKSVMLQIAATE 29 *.* $\quad: *: .: \quad:: *:: * \star::: * \star \star \star \quad$ *

HCTNI LEREAEERRGEKGRALSTRCQPLELAGLGFAELQDLCRQLHARVDKVDEERYDIEAKVTK 120 HFSTNI LEKEESRREAEKQNYLAEHCPPLHIPGS-MSEVQELCKQLHAKIDAAEEEKYDMEVRVQK 88

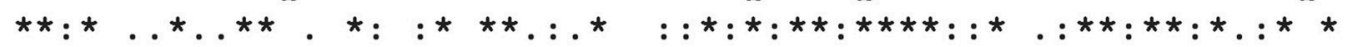

HCTNI NITEIADLTQKIFDLRGKFKRPTLRRVRISADAMMQALLGARAKESLDLRAHLKQVKKED 180 HFSTNI TSKELEDMNQKLFDLRGKFKRPPLRRVRMSADAMLKALLGSKHKVCMDLRANLKQVKKED 148

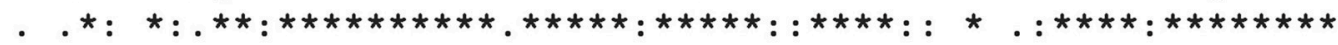
R204

HCTNI TEKEN--REVGDWRKNIDALSGMEGRKKKFES 210

HFSTNI TEKERDLRDVGDWRKNIEEKSGMEGRKKMFESES 182

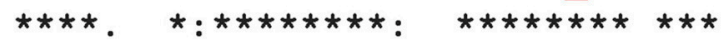

R174

FIGURE 1 | Primary structure of Human Cardiac Troponin I. (A) Alignment of the primary sequences of cardiac and fast skeletal Troponin I from different species. Some of the CTnl FHC mutations that occur within this region are indicated. The location of the R204 mutation is indicated. M, mouse; RB, rabbit; H, human; R, rat; B, bovine. (B) Alignment of the primary sequences of Human cardiac Troponin I (HCTNI) and Human fast skeletal Troponin I (HFSTNI). Human cardiac Troponin I (Swiss-Prot accession number: P19429). Human fast skeletal Troponin I (Swiss-Prot accession number: P48788). *Indicates identical amino acid.: indicates homology. 
A

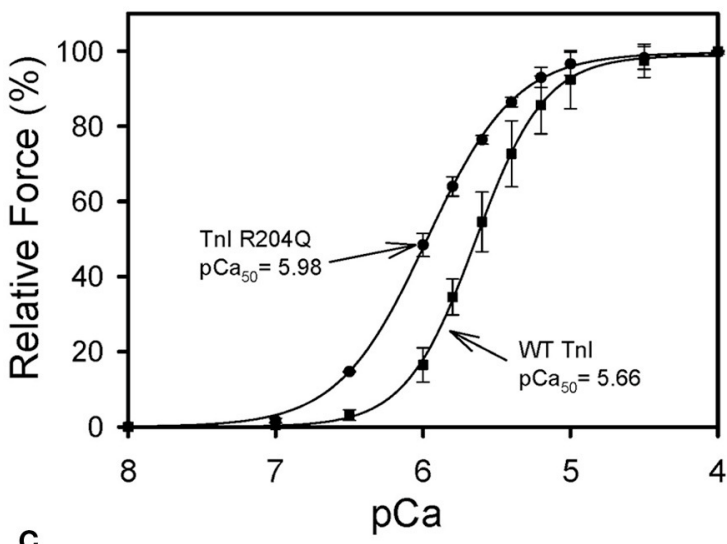

C

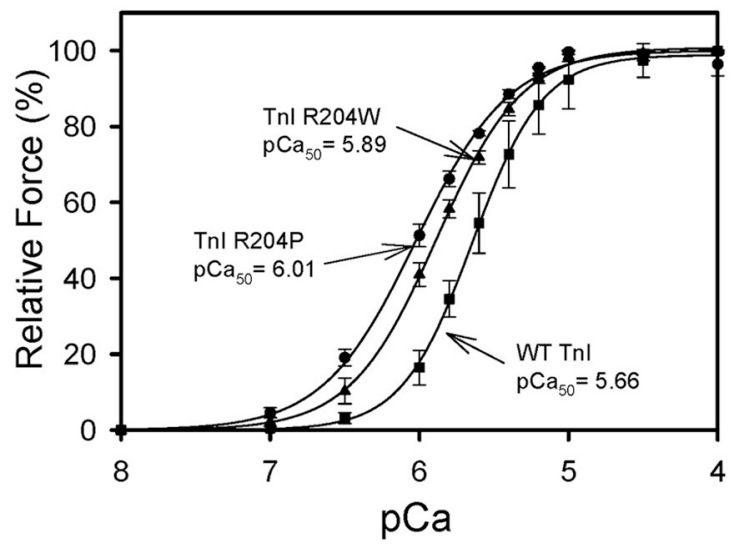

B
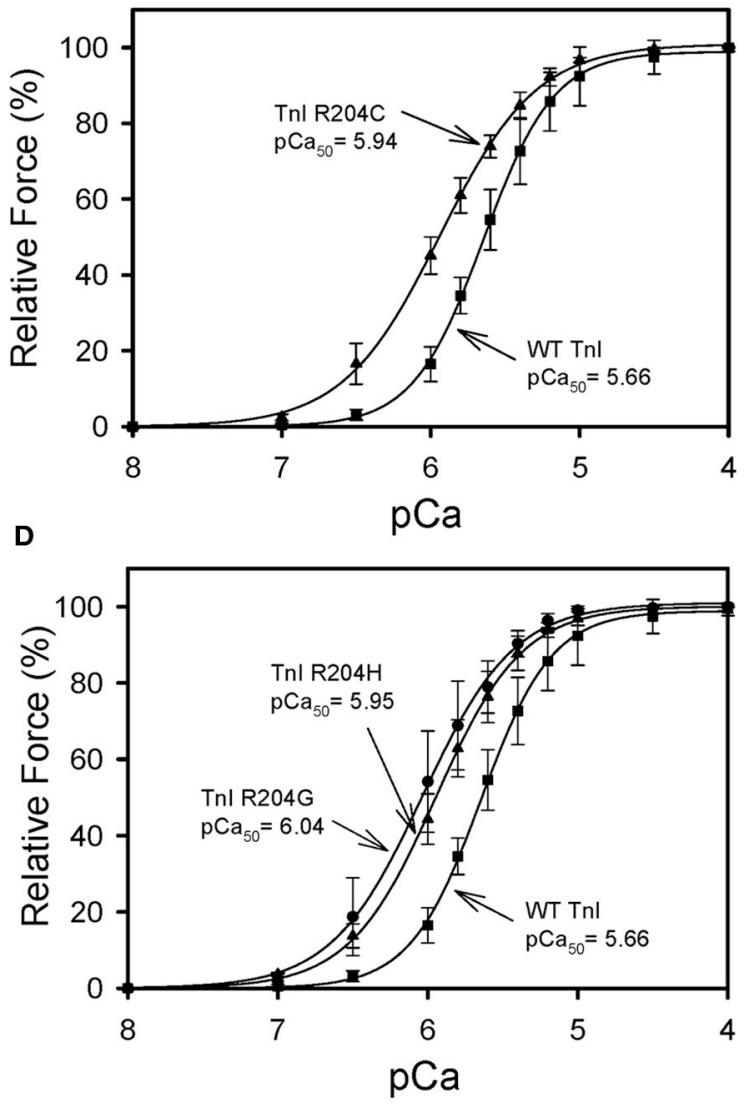

FIGURE 2 | Effect of mutations in Troponin I at R204 on the calcium sensitivity of force development. Each skinned muscle preparation was treated with $\mathrm{HcTnT}$ to displace the endogenous Tn complex and subsequently reconstituted with either HcTnl.HcTnC, or HcTnl R204 mutant. (A) Comparison of HcTnl.HcTnC and HcTnl R204Q.HCTnC. (B) Comparison of HcTnl.HcTnC and HcTnl R204C.HCTnC. (C) Comparison of HcTnl.HcTnC with HcTnl R204W.HcTnC or HcTnl R204P.HcTnC. (D) Comparison of HcTnl.HcTnC with HcTnl R204H.HcTnC or HcTnl R 204G.HcTnC. The Ca ${ }^{2+}$ dependence of force was measured in each preparation after reconstituting troponin. Each point is the average of 3 experiments and represents the mean \pm S.D. ${ }^{*} p<0.05$.

TABLE 1 | Effect of wild-type Human $\mathrm{cTnl}$ and mutants on the $\mathrm{Ca}^{2+}$ sensitivity of force development $\left(\mathrm{pCa}_{50}\right)$ and the Hill coefficient $\left(\mathrm{n}_{\mathrm{H}}\right)$ in skinned porcine cardiac muscle fibers.

\begin{tabular}{|c|c|c|c|c|}
\hline Human cTnl Mutant & $\mathrm{pCa}_{50}$ & Difference from Wild-type $\left(\Delta \mathrm{pCa}_{50}\right)$ & Hill coefficient $\left(n_{H}\right)$ & Number of experiments ( $n$ ) \\
\hline Wild-type (204R) & $5.66 \pm 0.07$ & - & $1.85 \pm 0.23$ & 3 \\
\hline $204 \mathrm{H}$ & $5.95 \pm 0.08^{\star}$ & 0.28 & $1.52 \pm 0.08^{*}$ & 3 \\
\hline $204 C$ & $5.94 \pm 0.07^{\star}$ & 0.27 & $1.35 \pm 0.07^{\star}$ & 3 \\
\hline $204 W$ & $5.89 \pm 0.03^{\star}$ & 0.22 & $1.50 \pm 0.03^{*}$ & 3 \\
\hline
\end{tabular}

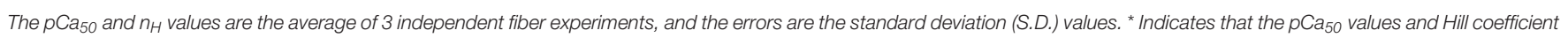
for the respective Tnl mutants are significantly different from wild-type $\mathrm{HcTnl}(p<0.05)$.

\section{Force Development and the $\mathrm{Ca}^{2+}$ Dependence of Force Development for fsTnl}

Rabbit fsTnI was studied in place of HfsTnI because it is the best characterized fast skeletal muscle system and the C-terminal half of rabbit fsTnI shows 100\% homology with HfsTnI. The homology between rabbit fsTnI and HfsTnI is $98.9 \%$. Under the conditions utilized, essentially all of the endogenous Tn complex was displaced following treatment with fsTnT. The amount of force development in the presence of very low concentration of $\mathrm{Ca}^{2+}$ (pCa 8.0) after fsTnT displacement is a measure of the 


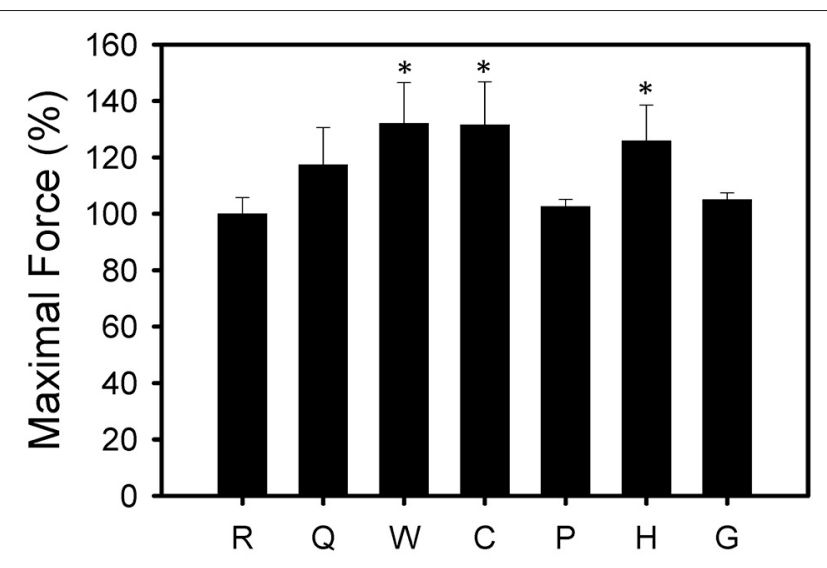

Cardiac Troponin I residue 204

FIGURE 3 | Effect of mutations in Troponin I at R204 on Maximal force. Each bar is the average of 3 experiments and represents the mean \pm S.D. Forces were normalized to the maximum force developed by each fiber after TnT treatment and HcTnl.HcTnC reconstitution. ${ }^{\star} P<0.05(n=3)$.

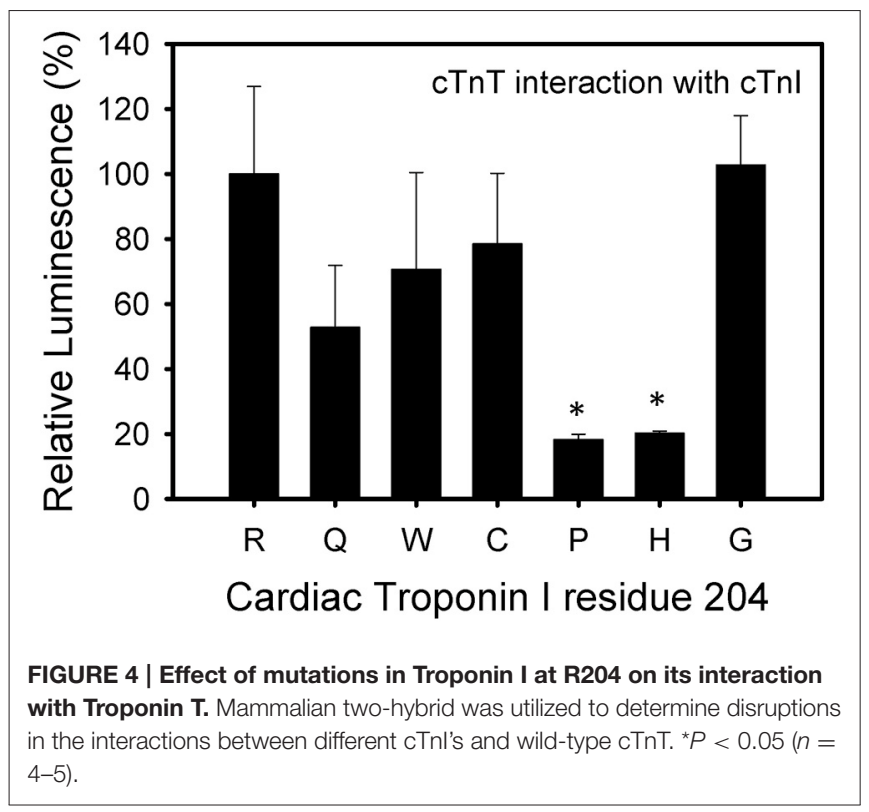

extent of displacement of endogenous fsTnI (referred to as the $\mathrm{Ca}^{2+}$ unregulated force). In the absence of fsTnI, fibers were unable to relax, as the inhibitory activity of $\mathrm{cTnI}$ is deficient. This measurement of $\mathrm{Ca}^{2+}$ unregulated force was utilized to ensure that all the skinned fibers are displaced to the same extent. All the fibers selected for these studies had a $\mathrm{Ca}^{2+}$ unregulated force of $>95 \%$.

After displacement and reconstitution, the maximal force obtained for each fiber was measured. This force was measured relative to the initial force of the skinned fibers before TnT displacement. An R174Q fsTnI mutation (associated with DA) increased the $\mathrm{Ca}^{2+}$ sensitivity of force development (Figure 6, Table 2). The results suggest that mutations associated with

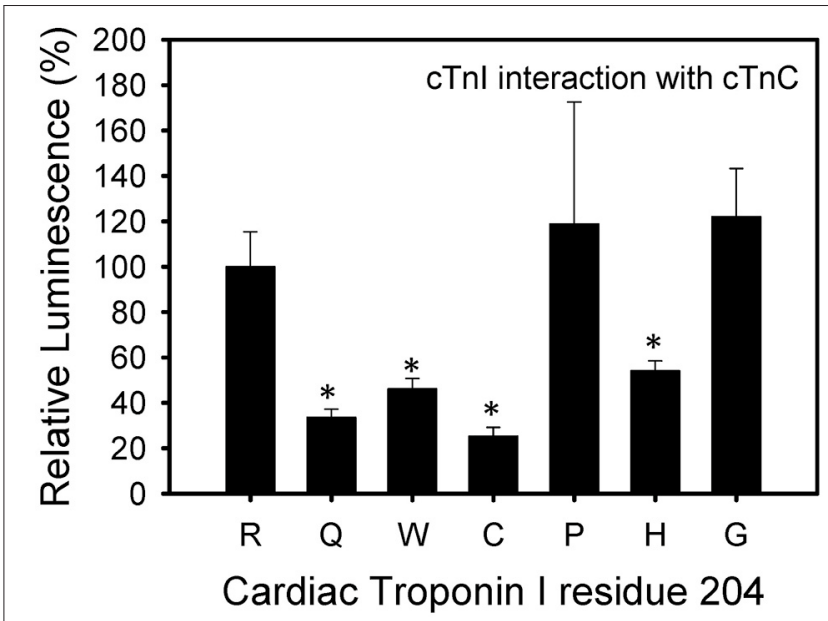

FIGURE 5 | Effect of mutations in Troponin I at R204 on its interaction with Troponin C. Mammalian two-hybrid was utilized to determine disruptions in the interactions between different cTnl's and wild-type cTnC. ${ }^{*} P$ $<0.05(n=4-5)$.

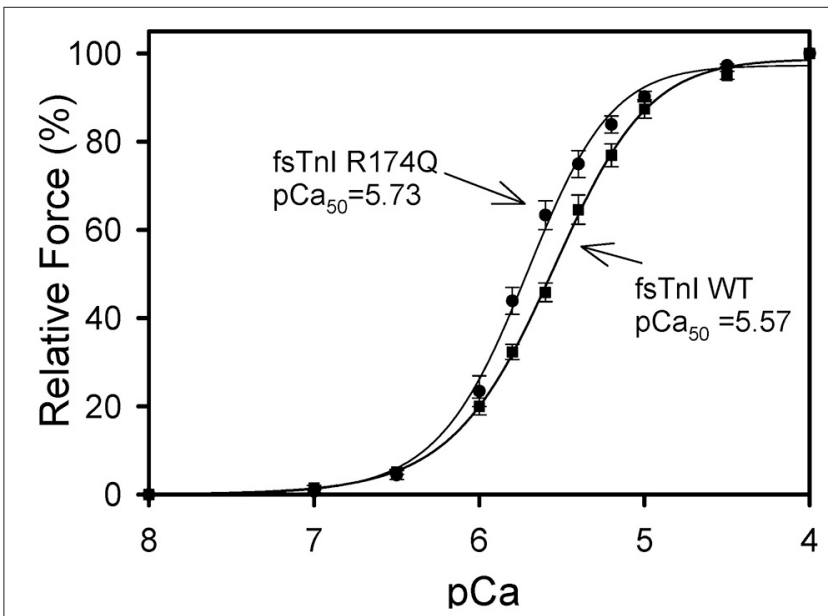

FIGURE 6 | Effect of Wild-type Fast Skeletal Tnl and Tnl R174Q on $\mathrm{Ca}^{2+}$-sensitivity of force development in skinned rabbit muscle fibers. After TnT treatment the fibers were reconstituted with Tnl-TnC complex.

Comparison of rabbit fibers after they were reconstituted with wild-type fsTnl or fsTnl R174Q in the presence of fsTnC. The $\mathrm{Ca}^{2+}$ dependence of force was measured in each preparation after reconstituting whole Tn. Each point is the average of 3-5 experiments and represents the mean $\pm S$.D.

significantly increased $\mathrm{Ca}^{2+}$ sensitivity of force development may be associated with HCM in cardiac tissue or DA in skeletal muscle. Unlike some of the cardiac R204 mutations which showed increased maximal force, the fsTnI R174Q mutant showed decreased maximal force relative to wild-type fsTnI (Figure 7).

\section{DISCUSSION}

Arginine residues in cTnI are associated with multiple mutations resulting in potentially different clinical phenotypes (Xu et al., 
TABLE 2 | Effect of wild-type Rabbit Fast Skeletal Tnl and TnI R174Q on the $\mathrm{Ca}^{2+}$-sensitivity of force development $\left(p C a_{50}\right)$ and the Hill coefficient $\left(n_{H}\right)$ in skinned fast skeletal muscle fibers.

\begin{tabular}{|c|c|c|c|c|}
\hline Fast skeletal Tnl & $\mathrm{pCa}_{50}$ & Difference from Wild-type $\left(\Delta \mathrm{pCa}_{50}\right)$ & Hill Coefficient $\left(n_{H}\right)$ & Number of experiments (n) \\
\hline Wild-type fsTnl & $5.57 \pm 0.01$ & - & $1.64 \pm 0.13$ & 3 \\
\hline fsTnl R174Q & $5.73 \pm 0.03^{*}$ & 0.16 & $1.65 \pm 0.10$ & 5 \\
\hline
\end{tabular}

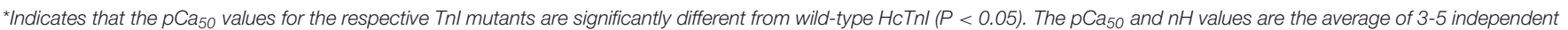
fiber experiments, and the errors are the standard deviation (S.D.) values. The average wild-type Tnl displacement was $97.6 \pm 2.4 \%$.

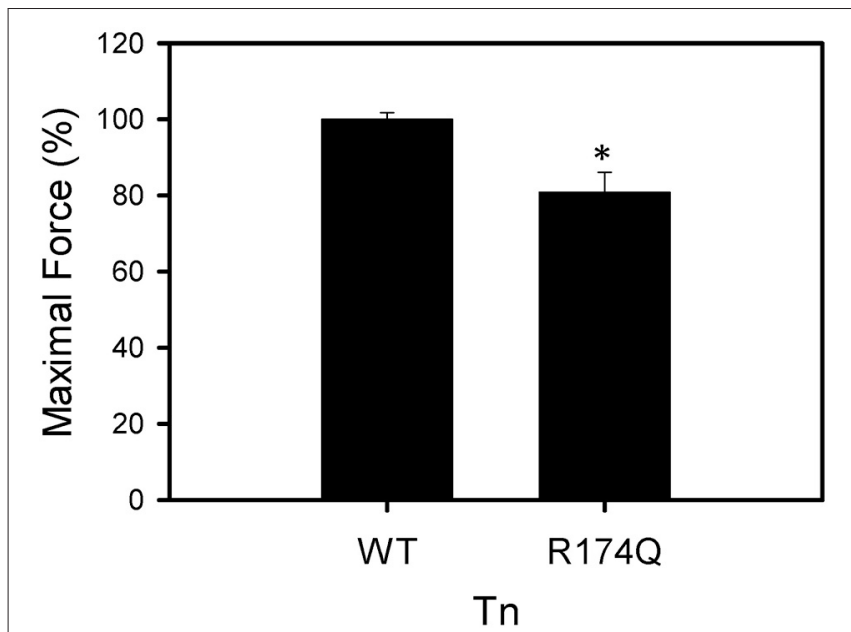

FIGURE 7 | Effect of Wild-type Fast Skeletal Tnl and Tnl R174Q on force development in skinned muscle fibers. Maximal force development of fibers after fsTnT displacement and fsTnl.fsTnC or fsTnl R174Q.fsTnC reconstitution. Each bar is the average of 3-5 experiments and represents the mean \pm S.D. ${ }^{\star} P<0.05$.

2010). Since two mutations (R204C and R204H) at residue 204 of cTnI are associated with FHC and most mutations associated with FHC show increased $\mathrm{Ca}^{2+}$-sensitivity of force development (Xu et al., 2010), we hypothesized that any mutations at cTnI R204 would cause significant increases in $\mathrm{Ca}^{2+}$-sensitivity of force development. To characterize the effects that different arginine mutations at the same residue would have on the physiological function of cTnI, six mutations at R204 (C, G, $\mathrm{H}, \mathrm{P}, \mathrm{Q}, \mathrm{W})$ were investigated in skinned fiber studies. All of the cTnI R204 mutations investigated displayed increased $\mathrm{Ca}^{2+}$ sensitivity of force development when compared to WT cTnI $\left(\Delta \mathrm{pCa}_{50}=0.22-0.37\right)$, similar to what is observed for most FHC mutations. These results suggest that the R204 residue is important in cTnI function and that increased $\mathrm{Ca}^{2+}$ sensitivity may be a major factor in HCM. The increase in myofilament sensitivity to $\mathrm{Ca}^{2+}$ is important since reduction in the $\mathrm{Ca}^{2+}$ sensitivity was found to prevent the development of HCM (Alves et al., 2014). Others have suggested that increased $\mathrm{Ca}^{2+}$-sensitivity is associated with HCM (Wei and Jin, 2015).

Certain regions of $\mathrm{cTnI}$ seem to be more important functionally than other regions (Mogensen et al., 2015; Wei and Jin, 2015; Meyer and Chase, 2016; Sheng and Jin, 2016).
It may be that any or most amino acid changes at certain TnI residues that occur in regions functionally important for regulating $\mathrm{Ca}^{2+}$-sensitivity would all be associated with increased $\mathrm{Ca}^{2+}$-sensitivity of force development. Transgenic mice expressing 9-17\% of a C-terminal truncated human cardiac TnI (residues 194-210 deleted, corresponding to residues 166182 of fsTnI), developed a phenotype of stunned myocardium (Murphy et al., 2000). These results suggest that the 194210 region of cTnI is important for cTnI function. The FHC mutations R204C and R204H both showed similar increases in $\mathrm{Ca}^{2+}$ sensitivity ( $\triangle \mathrm{pCa} 50$ values of 0.28 and 0.29 respectively). These two mutations also showed increased maximal force compared to WT cTnI. R204W also showed increased maximal force. The $\mathrm{R} 204 \mathrm{H}$ mutation was found to be associated with HCM in an Australian family and is associated with a poor prognosis (Doolan et al., 2005).

To determine how R204 mutations might disrupt proteinprotein interactions, a mammalian two-hybrid luciferase assay was used. Mutation of arginine to proline resulted in a significant reduction in functional interactions with both $c \operatorname{TnC}$ and $c \operatorname{TnT}$. The results suggest that binding of cTnI mutants (except R204G) to its binding partners is disrupted pending measurement of actual affinity constants. Interestingly, while the R204C mutation weakened the interaction of $\mathrm{TnI}$ with $\mathrm{TnC}$, it did not significantly affect the interaction of TnI with TnT. However, the cTnI $\mathrm{R} 204 \mathrm{H}$ mutation disrupted the interaction between cTnI and cTnT as well as between cTnI and cTnC, which is consistent with a previous report using a similar mammalian two hybrid assay (Doolan et al., 2005). Doolan et al. found evidence for altered interactions between TnI and either TnT, TnC, or both, for cTnI R162G, R162P, L194P, and R204H mutations. The previous finding that the cTnI R162G mutation reduced cTnI and $c$ TnT interaction while another mutation at the same cTnI residue, R162P, did not affect cTnI-cTnT interaction (Doolan et al., 2005) is consistent with our results which suggest that not all cTnI HCM mutations affect cTnI-cTnT mutations. Using the data obtained from the four mutations they investigated, Doolan et al. concluded that altered interactions in the Tn complex "increased severity of the disease." The replacement of cTnI arginine residue 204, a relatively large and charged amino acid, by glycine, the smallest amino acid, resulted in the largest increase in $\mathrm{Ca}^{2+}$-sensitivity of force development. The severity of the disease is likely to be more complicated due to altered interactions in the Tn complex since our R204G results suggest increased $\mathrm{Ca}^{2+}$-sensitivity of force development independent of any change in interactions with its Tn binding partners. 
Replacement of arginine with proline also resulted in a large increase in $\mathrm{Ca}^{2+}$ sensitivity of force development, which was significantly larger than the increase in $\mathrm{Ca}^{2+}$ sensitivity of force development observed for the R204W mutation $(p<0.05)$. This difference in $\mathrm{Ca}^{2+}$ sensitivity changes may be due to the size of the tryptophan residue, which is the largest amino acid and closer to the size of arginine than proline or glycine (Morris et al., 1992). Proline is also typically considered a structural disruptor of the protein secondary structure and its side chains are conformationally rigid, unlike glycine which can easily adopt many main chain conformations.

To extend the hypothesis that any mutation at cTnI R204 would cause significant increases in $\mathrm{Ca}^{2+}$ sensitivity of force development, we hypothesized that mutations at fsTnI R174, which occurs at an equivalent location to the cTnI R204 residue, would also cause significant increases in $\mathrm{Ca}^{2+}$-sensitivity of force development. We investigated the R174Q missense mutation associated with DA. The WT rabbit fsTnI consists of 182 residues, and the C-terminus region of fsTnI, which is affected by the R174Q mutation, is known to interact with $\mathrm{TnC}$ and actin. The fsTnI R174Q DA mutant showed increased $\mathrm{Ca}^{2+}$-sensitivity of force development, similar to what was observed for the cTnI R204Q mutation. Hence, the location of the R174Q DA mutation is likely to be physiologically important for the function of TnI. The R174Q was previously investigated by another group that showed a greater increase in $\mathrm{Ca}^{2+}$-sensitivity of force development $\left(\Delta \mathrm{pCa}_{50}=0.36\right)$ and no change in maximal force (Robinson et al., 2007). The differences from what we observed may be due to species of cTnI utilized, as Robinson et al. utilized human fsTnI in muscle fibers in rabbit skeletal muscle fibers while we utilized rabbit fsTnI in rabbit skeletal muscle fibers (Robinson et al., 2007). However, in both cases the increase in $\mathrm{Ca}^{2+}$-sensitivity of force development relative to WT fsTnI was significant.

DA occurs in about 1 in 3000 children, and other fsTnI mutations have been found to be associated with DA2B (fsTnI R156ter, K175del, and K176del mutations) (Kimber et al., 2012). Patients with DA2B have contractures which are present at birth, suggesting impaired relaxation of muscle fibers. A likely possibility is that the higher $\mathrm{Ca}^{2+}$-sensitivity of force development in fibers containing DA2B mutations affects muscle contracture. These results, as well as two other reports that showed increased $\mathrm{Ca}^{2+}$-sensitivity with DA2B mutations (Robinson et al., 2007; Mokbel et al., 2013), all suggest that joint and muscle contractures may be caused by prolonged muscle hypercontraction due to increased myofibrillar calcium sensitivity and that treatments that reduce skeletal muscle $\mathrm{Ca}^{2+}$ sensitivity may be beneficial to patients with DA2B.

Overall, these results suggest that different amino acids at the same site on cTnI could affect thin filament interactions differentially. While significant impairment in the interactions of cTnI with TnT or TnC may be enough to cause significant changes in $\mathrm{Ca}^{2+}$-sensitivity of force development, impairment in these interactions is not a requirement for altering the $\mathrm{Ca}^{2+}$. sensitivity as our study showed that R204G had the largest increase in $\mathrm{Ca}^{2+}$-sensitivity yet demonstrated maximal tension and cTnI:TnT/TnC interactions comparable to wild-type levels. While R204G did not show any altered interactions between cTnI and TnC or TnT it is possible that altered interactions between TnI R204G and actin may occur. Unfortunately, we were unable to get cTnI:actin two hybrid studies to work well. It may also be possible that the R204 mutations are affecting thin and thick filament interactions by disturbing tropomyosin displacement. If the large increase in $\mathrm{Ca}^{2+}$-sensitivity of force development observed with these mutations is associated with the poor prognosis, then many other R204 mutations that may be discovered in the population are likely to have poor prognoses.

\section{ETHICS STATEMENT}

This study was exempt from this requirement because the tissues were obtained from the University of California, Davis Veterinary School Slaughterhouse. The Veterinary School Slaughterhouse routinely sacrifices animals for teaching purposes and sells the meat from these animals to the public. A qualified researcher can pay a small fee $(\$ 10)$ and get heart tissue or some skeletal muscle for research from these animals that have already been sacrificed.

\section{AUTHOR CONTRIBUTIONS}

AG: design of work, experiments, acquisition, interpretation of data, drafting of work, final approval, agreement to be accountable for all aspects of work. SN: acquisition, interpretation of data, revising, final approval, agreement to be accountable for all aspects of work. RS: acquisition, revising, final approval, agreement to be accountable for all aspects of work. SD: acquisition, revising, final approval, agreement to be accountable for all aspects of work. ZC: interpretation of data, revising, final approval, agreement to be accountable for all aspects of work.

\section{FUNDING}

This research was supported by a UC Davis funds and a Hellman Fellowship (AG).

\section{ACKNOWLEDGMENTS}

We would like to thank Dr. Jingsheng Liang (University of Miami) for help with the fiber studies. 


\section{REFERENCES}

Alves, M. L., Dias, F. A., Gaffin, R. D., Simon, J. N., Montminy, E. M., Biesiadecki, B. J., et al. (2014). Desensitization of myofilaments to $\mathrm{Ca}^{2+}$ as a therapeutic target for hypertrophic cardiomyopathy with mutations in thin filament proteins. Circ. Cardiovasc. Genet. 7, 132-143. doi: 10.1161/CIRCGENETICS.113.000324

Bonne, G., Carrier, L., Bercovici, J., Cruaud, C., Richard, P., Hainque, B., et al. (1995). Cardiac myosin binding protein-C gene splice acceptor site mutation is associated with familial hypertrophic cardiomyopathy. Nat. Genet. 11, 438-440. doi: $10.1038 / \mathrm{ng} 1295-438$

Doolan, A., Tebo, M., Ingles, J., Nguyen, L., Tsoutsman, T., Lam, L., et al. (2005). Cardiac troponin I mutations in Australian families with hypertrophic cardiomyopathy: clinical, genetic and functional consequences. J. Mol. Cell. Cardiol. 38, 387-393. doi: 10.1016/j.yjmcc.2004.12.006

Geisterfer-Lowrance, A. A., Kass, S., Tanigawa, G., Vosberg, H. P., McKenna, W., Seidman, C. E., et al. (1990). A molecular basis for familial hypertrophic cardiomyopathy: a beta cardiac myosin heavy chain gene missense mutation. Cell 62, 999-1006. doi: 10.1016/0092-8674(90)90274-I

Gilda, J. E., Xu, Q., Martinez, M. E., Nguyen, S. T., Chase, P. B., and Gomes, A. V. (2016). The functional significance of the last 5 residues of the Cterminus of cardiac troponin I. Arch. Biochem. Biophys. 601, 88-96. doi: 10.1016/j.abb.2016.02.023

Gomes, A. V., Guzman, G., Zhao, J., and Potter, J. D. (2002). Cardiac troponin $\mathrm{T}$ isoforms affect the $\mathrm{Ca}^{2+}$ sensitivity and inhibition of force development. Insights into the role of troponin T isoforms in the heart. J. Biol. Chem. 277, 35341-35349. doi: 10.1074/jbc.M204118200

Gomes, A. V., Harada, K., and Potter, J. D. (2005a). A mutation in the N-terminus of troponin I that is associated with hypertrophic cardiomyopathy affects the $\mathrm{Ca}^{(2+)}$-sensitivity, phosphorylation kinetics and proteolytic susceptibility of troponin. J. Mol. Cell. Cardiol. 39, 754-765. doi: 10.1016/j.yjmcc.2005. 05.013

Gomes, A. V., Liang, J., and Potter, J. D. (2005b). Mutations in human cardiac troponin I that are associated with restrictive cardiomyopathy affect basal ATPase activity and the calcium sensitivity of force development. J. Biol. Chem. 280, 30909-30915. doi: 10.1074/jbc.M500287200

Gomes, A. V., and Potter, J. D. (2004). Cellular and molecular aspects of familial hypertrophic cardiomyopathy caused by mutations in the cardiac troponin I gene. Mol. Cell. Biochem. 263, 99-114. doi: 10.1023/B:MCBI.0000041852.42291.aa

Gomes, A. V., Venkatraman, G., Davis, J. P., Tikunova, S. B., Engel, P., Solaro, R. J., et al. (2004). Cardiac troponin $\mathrm{T}$ isoforms affect the $\mathrm{Ca}(2+)$ sensitivity of force development in the presence of slow skeletal troponin I: insights into the role of troponin T isoforms in the fetal heart. J. Biol. Chem. 279, 49579-49587. doi: 10.1074/jbc.M407340200

Hatakenaka, M., and Ohtsuki, I. (1992). Effect of removal and reconstitution of troponins $\mathrm{C}$ and $\mathrm{I}$ on the $\mathrm{Ca}(2+)$ - activated tension development of single glycerinated rabbit skeletal muscle fibers. Eur. J. Biochem. 205, 985-993. doi: 10.1111/j.1432-1033.1992.tb16865.x

Hoffmann, B., Schmidt-Traub, H., Perrot, A., Osterziel, K. J., and Gessner, R. (2001). First mutation in cardiac troponin C, L29Q, in a patient with hypertrophic cardiomyopathy. Hum. Mutat. 17, 524. doi: 10.1002/hu mu.1143

Kimber, E., Tajsharghi, H., Kroksmark, A. K., Oldfors, A., and Tulinius, M. (2012). Distal arthrogryposis: clinical and genetic findings. Acta Paediatr. 101, 877-887. doi: $10.1111 / j .1651-2227.2012 .02708 . x$

Kimura, A., Harada, H., Park, J. E., Nishi, H., Satoh, M., Takahashi, M., et al. (1997). Mutations in the cardiac troponin I gene associated with hypertrophic cardiomyopathy. Nat. Genet. 16, 379-382. doi: 10.1038/ng0897-379

Kokado, H., Shimizu, M., Yoshio, H., Ino, H., Okeie, K., Emoto, Y., et al. (2000). Clinical features of hypertrophic cardiomyopathy caused by a Lys 183 deletion mutation in the cardiac troponin I gene. Circulation 102, 663-669. doi: 10.1161/01.CIR.102.6.663

Lang, R., Gomes, A. V., Zhao, J., Housmans, P. R., Miller, T., and Potter, J. D. (2002). Functional analysis of a troponin I (Arg145Gly) mutation associated with familial hypertrophic cardiomyopathy. J. Biol. Chem. 277, 11670-11678. doi: 10.1074/jbc.M108912200
Meyer, N. L., and Chase, P. B. (2016). Role of cardiac troponin I carboxy terminal mobile domain and linker sequence in regulating cardiac contraction. Arch. Biochem. Biophys. 601, 80-87. doi: 10.1016/j.abb.2016. 03.010

Mogensen, J., Hey, T., and Lambrecht, S. (2015). A systematic review of phenotypic features associated with cardiac Troponin I mutations in hereditary Cardiomyopathies. Can. J. Cardiol. 31, 1377-1385. doi: 10.1016/j.cjca.2015.06.015

Mogensen, J., Klausen, I. C., Pedersen, A. K., Egeblad, H., Bross, P., Kruse, T. A., et al. (1999). Alpha-cardiac actin is a novel disease gene in familial hypertrophic cardiomyopathy. J. Clin. Invest. 103, R39-R43. doi: 10.1172/ JCI6460

Mokbel, N., Ilkovski, B., Kreissl, M., Memo, M., Jeffries, C. M., Marttila, M., et al. (2013). K7del is a common TPM2 gene mutation associated with nemaline myopathy and raised myofibre calcium sensitivity. Brain 136(Pt 2), 494-507. doi: 10.1093/brain/aws348

Mörner, S., Richard, P., Kazzam, E., Hainque, B., Schwartz, K., and Waldenström, A. (2000). Deletion in the cardiac troponin I gene in a family from northern Sweden with hypertrophic cardiomyopathy. J. Mol. Cell. Cardiol. 32, 521-525. doi: 10.1006/jmcc.1999.1099

Morris, A. L., MacArthur, M. W., Hutchinson, E. G., and Thornton, J. M. (1992). Stereochemical quality of protein structure coordinates. Proteins 12, 345-364. doi: $10.1002 /$ prot. 340120407

Murphy, A. M., Kögler, H., Georgakopoulos, D., McDonough, J. L., Kass, D. A., Van Eyk, J. E., and Marbán, E. (2000). Transgenic mouse model of stunned myocardium. Science 287, 488-491. doi: 10.1126/science.287. 5452.488

Niimura, H., Patton, K. K., McKenna, W. J., Soults, J., Maron, B. J., Seidman, J. G., et al. (2002). Sarcomere protein gene mutations in hypertrophic cardiomyopathy of the elderly. Circulation 105, 446-451. doi: 10.1161/hc0402.102990

O'Mahony, C., and Elliott, P. M. (2014). Prevention of sudden cardiac death in hypertrophic cardiomyopathy. Heart 100, 254-260. doi: 10.1136/heartjnl-2012301996

Pan, B. S., and Potter, J. D. (1992). Two genetically expressed troponin T fragments representing alpha and beta isoforms exhibit functional differences. J. Biol. Chem. 267, 23052-23056.

Poetter, K., Jiang, H., Hassanzadeh, S., Master, S. R., Chang, A., Dalakas, M. C., et al. (1996). Mutations in either the essential or regulatory light chains of myosin are associated with a rare myopathy in human heart and skeletal muscle. Nat. Genet. 13, 63-69. doi: 10.1038/ng0596-63

Robinson, P., Lipscomb, S., Preston, L. C., Altin, E., Watkins, H., Ashley, C. C., et al. (2007). Mutations in fast skeletal troponin I, troponin T, and beta-tropomyosin that cause distal arthrogryposis all increase contractile function. FASEB J. 21, 896-905. doi: 10.1096/fj.06-6899com

Satoh, M., Takahashi, M., Sakamoto, T., Hiroe, M., Marumo, F., and Kimura, A. (1999). Structural analysis of the titin gene in hypertrophic cardiomyopathy: identification of a novel disease gene. Biochem. Biophys. Res. Commun. 262, 411-417. doi: 10.1006/bbrc.1999.1221

Sheng, J. J., and Jin, J. P. (2016). TNNI1, TNNI2 and TNNI3: evolution, regulation, and protein structure-function relationships. Gene 576(1 Pt 3), 385-394. doi: 10.1016/j.gene.2015.10.052

Sheng, Z., Pan, B. S., Miller, T. E., and Potter, J. D. (1992). Isolation, expression, and mutation of a rabbit skeletal muscle cDNA clone for troponin I. The role of the $\mathrm{NH} 2$ terminus of fast skeletal muscle troponin I in its biological activity. J. Biol. Chem. 267, 25407-25413.

Szczesna, D., Zhang, R., Zhao, J., Jones, M., Guzman, G., and Potter, J. D. (2000). Altered regulation of cardiac muscle contraction by troponin $\mathrm{T}$ mutations that cause familial hypertrophic cardiomyopathy. J. Biol. Chem. 275, 624-630. doi: $10.1074 /$ jbc.275.1.624

Thierfelder, L., Watkins, H., MacRae, C., Lamas, R., McKenna, W., Vosberg, H. P., et al. (1994). Alpha-tropomyosin and cardiac troponin T mutations cause familial hypertrophic cardiomyopathy: a disease of the sarcomere. Cell 77, 701-712. doi: 10.1016/0092-8674(94)90054-X

Tobacman, L. S. (1996). Thin filament-mediated regulation of cardiac contraction. Annu. Rev. Physiol. 58, 447-481. doi: 10.1146/annurev.ph.58.030196. 002311 
Watkins, H., Conner, D., Thierfelder, L., Jarcho, J. A., MacRae, C., McKenna, W. J., et al. (1995). Mutations in the cardiac myosin binding protein-C gene on chromosome 11 cause familial hypertrophic cardiomyopathy. Nat. Genet. 11, 434-437. doi: 10.1038/ng1295-434

Wei, H., and Jin, J. P. (2015). NH2-terminal truncations of cardiac troponin I and cardiac troponin $\mathrm{T}$ produce distinct effects on contractility and calcium homeostasis in adult cardiomyocytes. Am. J. Physiol. Cell Physiol. 308, C397C404. doi: 10.1152/ajpcell.00358.2014

Westfall, M. V., and Metzger, J. M. (2001). Troponin I isoforms and chimeras: tuning the N molecular switch of cardiac contraction. News Physiol. Sci. 16, $278-281$.

Xu, Q., Dewey, S., Nguyen, S., and Gomes, A. V. (2010). Malignant and benign mutations in familial cardiomyopathies: insights into mutations linked to complex cardiovascular phenotypes. J. Mol. Cell. Cardiol. 48, 899-909. doi: 10.1016/j.yjmcc.2010.03.005

Zhang, R., Zhao, J., Mandveno, A., and Potter, J. D. (1995). Cardiac troponin I phosphorylation increases the rate of cardiac muscle relaxation. Circ. Res. 76, 1028-1035. doi: 10.1161/01.RES.76. 6.1028
Zot, A. S., and Potter, J. D. (1987). Structural aspects of troponintropomyosin regulation of skeletal muscle contraction. Annu. Rev. Biophys. Biophys. Chem. 16, 535-559. doi: 10.1146/annurev.bb.16.060187. 002535

Conflict of Interest Statement: The authors declare that the research was conducted in the absence of any commercial or financial relationships that could be construed as a potential conflict of interest.

The reviewer HSH and handling Editor declared their shared affiliation, and the handling Editor states that the process nevertheless met the standards of a fair and objective review.

Copyright (c) 2016 Nguyen, Siu, Dewey, Cui and Gomes. This is an open-access article distributed under the terms of the Creative Commons Attribution License (CC BY). The use, distribution or reproduction in other forums is permitted, provided the original author(s) or licensor are credited and that the original publication in this journal is cited, in accordance with accepted academic practice. No use, distribution or reproduction is permitted which does not comply with these terms. 